\title{
A MEASUREMENT MODEL TO DETERMINE THE ABILITY OF A REGION TO PROVIDE CLAEN WATER Jing Tian
}

Department of Mathematics and Physics, North China Electric Power University, Baoding 071003, China; 15932135705@163.com

\begin{abstract}
Freshwater resources are the basis for the survival of many organisms, especially for human. However, freshwater resources are gradually scarce. To provide people with more freshwater, the first thing we need to do is to determine the ability of a region to provide clean water to meet the needs of its population. In this paper, in order to determine the ability of a region to provide clean water to meet the needs of its population, we will build the measurement model. In this model, we combine the multivariable linear regression model with the $\operatorname{DGM}(2,1)$ model of Grey Prediction. At the same time, the dynamic nature of the factors that affect both supply and demand are considered in the modeling process.
\end{abstract}

Keywords: multivariable linear regression, Grey Prediction, $\operatorname{DGM}(2,1)$

\section{Introduction}

Water resources are abundant in the world, but the proportion of freshwater is very small(Fig.1). However, the demand for freshwater is very large: agricultural, industrial and residential purposes, etc. And because of the physical and economic scarcity, water scarcity has become a critical issue. So it is important and necessary to help humans improve access to clean, fresh water.

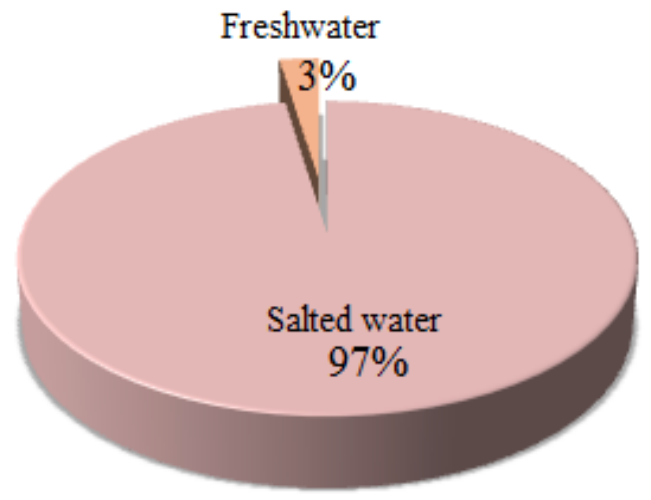

Fig.1 Composition of water resources.

Therefore, we develop a model, which provides a measure of the ability of a region to provide clean water to meet the needs of its population. 


\section{The measurement model}

\subsection{Problem Analysis}

According to the requirements in Task 1, we need a measurement model of the ability of a region to provide clean water to meet the needs of its population. And in our modeling process, we consider the dynamic nature of the factors that affect both supply and demand.

\subsection{Assumptions}

- The total supply of clean water in the area is only related to industrial water, agricultural water, residential water and rainfall.

- Per capita water demand to meet the normal life of the area in the next 15 years maintains the same

- The total of clean water supply is all used, without the phenomenon of waste.

- The total demand for clean water in the area is only related to the size of the population. The greater the population, the greater the total demand for water.

\subsection{Symbols and Descriptions}

Table 1 Symbols and Descriptions

\begin{tabular}{cl}
\hline Symbols & Descriptions \\
\hline $\boldsymbol{y}_{\mathbf{1}}$ & The annual total supply of clean water in the region. \\
$\begin{array}{c}\text { 错误! 未 } \\
\text { 找到引 }\end{array}$ & The annual total demand of clean water in the region. \\
用源。 & \\
$\boldsymbol{x}_{\mathbf{1}}$ & The annual total supply of industrial water in the region. \\
$\boldsymbol{x}_{\mathbf{2}}$ & The annual total supply of agricultural water in the region. \\
$\boldsymbol{x}_{\mathbf{3}}$ & The annual total supply of residential water in the region. \\
$\boldsymbol{x}_{\mathbf{4}}$ & The annual total rainfall in the region. \\
$m$ & Annual per capita water demand to meet the normal life in the region \\
$n$ & The total population of the year in this region. \\
\hline
\end{tabular}

\subsection{The measurement model}

The basic model has two parts: a multivariable linear regression model and a $\operatorname{DGM}(2,1)$ model of Grey Prediction. The first model is used to analyze the relation between water supply and its influence factors. And the second can predict the water of industry (错误! 未找到引用源。1), agriculture (错误! 未找到引用源。2), residents (错误! 未找到引用源。3) and rainfall (错误! 未找 到引用源。 4). Then, we compare the total of water supply with the total of water demand to determine the ability of water supply in this region.

Step 1: Modeling process

Sub-step 1: Multivariable linear regression

According to the date in recent years, we obtain a regression equation by the multivariable linear regression model:

$$
y_{1}=a_{1} x_{1}+a_{2} x_{2}+a_{3} x_{3}+a_{4} x_{4}+b
$$


Then, using the $\operatorname{DGM}(2,1)$ model, we get the year's $x_{1}, x_{2}, x_{3}, x_{4}$. Based on the above equation, we get the year's water supply 错误! 未找到引用源。 1 .

According to the population and annual per capita water consumption, we can know the water demand is

$$
\text { 错误!未找到引用源。 }
$$

Sub-step 2: Grey Prediction

Based on the data, we use the $\operatorname{DGM}(2,1)$ model in the Grey Prediction to predict the per capita water consumption per year in the chosen region or country.

Set the original sequence as:

$$
z^{(0)}=\left(z^{(0)}(1), z^{(0)}(2), \cdots, z^{(0)}(6)\right)
$$

Sequentially accumulated generate sequence $x^{(1)}$ and sequentially subtracting generate sequence $\alpha^{(1)} t^{(0)}$,respectively as

$$
\begin{aligned}
z^{(1)} & =\left(z^{(1)}(1), z^{(1)}(2), \cdots, z^{(1)}(6)\right) \\
\alpha^{(1)} z^{(0)} & =\left(\alpha^{(1)} z^{(0)}(1), \alpha^{(1)} z^{(0)}(2), \cdots, \alpha^{(1)} z^{(0)}(6)\right)
\end{aligned}
$$

in the two sequences, $z^{(1)}(k)=\sum_{i=1}^{k} z^{(0)}(i) ; k=1,2, \cdots, 6$

$$
\alpha^{(1)} z^{(1)}(k)=\sum_{i=1}^{k} z^{(0)}(k)=z^{(0)}(k)-z^{(0)}(k-1) ; k=2,3, \cdots, 6 .
$$

The $\operatorname{DGM}(2,1)$ model is

$$
\alpha^{(1)} z^{(0)}(k)+a z^{(0)}(k)=b
$$

The Whitenization Equation of the $\operatorname{DGM}(2,1)$ model is

$$
\frac{d^{2} z^{(1)}}{d t}+a \frac{d z^{(1)}}{d t}=b
$$

Based on the above equations, we obtain the prediction equation

\section{Step 2: Comparison}

If 错误!未找到引用源。 $1 \geq y_{2}$, the ability of water supply is enough to meet the water demand in this region; else, the ability of water supply can't meet the demand for water in this region.

\section{Strengths and weaknesses}

\subsection{Strengths}

- Through the measurement model, we can compare the water supply with the water demand. Thus, the ability of a region to provide clean water to meet the needs of its population can be determined. 
- The measurement model is easy to understand and obtained. So we solve the problem with a simple model.

- Grey Prediction doesn't need a lot of data to solve the problem, which has the little historical data, the low integrity of the sequence and the low reliability.

\subsection{Weaknesses}

- In reality, the supply and demand of water are related to many factors. Therefore, the standard of water use may change with time

- Grey Prediction only suitable for medium and short term prediction.

\section{Conclusions}

This paper solves the problem of water scarcity and puts forward the measurement model to determine the ability of a region to provide clean, fresh water. For a region, we can use this model to discuss its reasons of water scarcity. Through analysis, we make effective recommendations to save and protect water.

However, there is still much to be done in the future. On one hand, in our model, we only consider that the total supply of water is related to industry, agriculture, residential life and rainfall. To be honest, it is incorrect to some extent. Therefore, we can consider many other factors in the future, which will make the models more accurate and more fit for real life. On the other hand, in the modeling process, we can contact the related water institutions. Thus, we can not only improve our models but also improve the feasibility of the solutions.

\section{References}

[1] Shangyou Feng, Introduction to sustainable utilization and management of water resources, Science Press, 2002, p.229-240.

[2] Information on http://worldwater.org.

[3] Information on www.unep.org. 